\title{
Editorial
}

\section{Nanosized Analytical Devices for Biomedical and Pharmaceutical Applications}

Analytical methods provide all the other disciplines such as biology, pharmaceutical chemistry, pharmaceutical technology, biotechnology and many other fields with data on the character and amount of chemical species contained in natural and manmade objects and on the distribution of these species in space and time. Within the last decade, very rapid changes have taken place in analytical sciences, nanomaterials and pharmaceutical industry activities. Among these, the impact of developments in analytical and computer sciences, nanotechnology and medical sciences have also added tremendous challenges to pharmaceuticals and their applications. We all know what we are facing new challenges everyday in our mutual applications. Developments in nanoscience and nanotechnology have already had a far-reaching impact in traditional analytical chemistry, opening a new era in analytical methodology and instrumentation. The new nano-sensors allow biological assays with a higher sensitivity and throughput than those for conventional analytical methods.

New drugs coming from biotechnology, and their dosage forms, like targeted drugs, may produce new analytical problems in the future. The various analytical methods that permit the determination of drugs in their dosage forms, raw material, biological media etc, and also their metabolites to be separated, identified and quantitatively assayed are briefly reviewed at nano level amount. The current significance of separation, electrochemical, spectrophotometric and other methods is highlighted, as well as the limits of trace assays. The sensitivity of the analytical assay has a direct impact on the validity of the pharmacokinetic model which is built up from plasma concentration data. The precision and accuracy of the assay is also important, and it is not always straightforwardly estimated. A new significant parameter is the speed of analysis, and the resulting massive production of analytical data. In addition to other analytical methods, the use of electrochemical methods to gain key information about drug molecules and their mechanism of action is getting one of the important ways in drug discovery. Although electrochemical data do not give absolute relationship with biological activity, due to in vitro conditions it is still possible to consider that the oxidation mechanisms taking place at the electrode and in the body share related principles as a result of the existing similarities between electrochemical and biological reactions.

From the viewpoints mentioned above, the title "Nanosized Analytical Devices for Biomedical and Pharmaceutical Applications" of this special Hot Topic issue of The Open Chemical and Biomedical Methods Journal was chosen so as to ask analytical chemists and pharmaceutical scientists to appreciate their great roles in biomedical and pharmaceutical science. This special issue features $\mathbf{5}$ reviews and $\mathbf{7}$ original research papers. In this issue resent developments of separation techniques such as capillary electrophoresis, liquid chromatography, high throughput screening methodologies, chiral analysis and spectrophotometric methods and their applications, current progresses in DNA biosensors, nanosensors, electroanalytical methods, new electrode materials such as carbon nanotubes, boron doped diamond, etc., are presented.

Specifically, contributed articles in this special Hot Topic issue of The Open Chemical and Biomedical Methods Journal include the review paper by Radi which describes the results regarding the voltammetric determination of micromolar, submicromolar and nanomolar concentrations of various fluoroquinolones antibacterial agents with use of different electrodes. Tzanavaras presented and discussed automated sequential injection based methods for the determination of pharmaceutically and biologically important thiols, namely cysteine, $\mathrm{N}$-acetylcysteine, penicillamine, glutathione and captopril in his review paper. Aboul-Enein et al. focused on sample treatment, applications, optimization, detection, mechanisms of chiral resolution and future perspectives of $\mathrm{CE}$ in chiral resolution of xenobiotics in their review article. Dogan-Topal et al. described both the underlying theory and the practical guidance needed to apply square wave techniques and also provided a wide collection of data for the description of diverse tendencies that characterize several electrochemical reactions analyzed by square wave voltammetry. In his second review, Radi described the cyclodextrins which are related with electrochemistry of and cyclodextrin (CD) inclusion complexes with different pharmaceutical compounds. In research paper by Stefan-van-Staden et al. enantioselective, potentiometric membrane electrodes based on $\alpha$-, $\beta$ - and $\gamma$-cyclodextrins as chiral selectors were proposed for the assay of $S$-deprenyl. Oros et al. was worked on the measurement of the growth of some Trichoderma species on media containing CDs as unique carbon source, and the application of 3D-PCA for the assessment of the relationships among the capacity of Trichoderma species to utilize CDs. In paper by Sotomayor et al. a biomimetic sensor as a promising new analytical method for determination of norfloxacin in pharmaceuticals was reported. Uslu et al. was developed a selective and sensitive assay for the determination of ciprofloxacin using glassy carbon electrode. Goyal et al. was proposed a voltammetric sensor based on the nano $\mathrm{TiO}_{2}$ powder modified glassy carbon electrode for determination of dopamine. Aboul-Enein et al. was developed a rapid and sensitive spectrophotometric method for the determination of diosmin in their research study. Similarly, in her paper Onay-Besikci described the model for the radioactive measurements as an indicator of metabolic fluxes in myocytes.

This special Hot Topic issue of The Open Chemical and Biomedical Methods Journal is multidisciplinary. Also recent developments of application, evaluation and validation of analytical methods are focused by key topics in drug developments and analysis by assessment of the distinguished authors of this special issue. Thus, I expect this special Hot Topic issue will assist readers to find out new information and to encourage them to contribute more to recent development on drug development 
and its analysis using different methods. I hope that the reader will find a number of topics of interest, and that additional new ideas will emerge from this special issue.

I would like to thank to all of the authors for their excellent contributions, and the Editor in Chief of The Open Chemical and Biomedical Methods Journal for his kind invitation to act as Guest Editor for this special issue.

Sibel A. Ozkan

(Guest Editor)

Faculty of Pharmacy

Department of Analytical Chemistry

Ankara University

06100, Tandogan

Ankara

Turkey

E-mails: ozkan@pharmacy.ankara.edu.tr, sibelaysil@gmail.com

(C) Sibel A. Ozkan; Licensee Bentham Open.

This is an open access article licensed under the terms of the Creative Commons Attribution Non-Commercial License (http://creativecommons.org/licenses/by$\mathrm{nc} / 3.0 /$ ) which permits unrestricted, non-commercial use, distribution and reproduction in any medium, provided the work is properly cited. 